\title{
Association of orexin/hypocretin receptor gene (HCRTR1) with reward sensitivity, and interaction with gender
}

\author{
Aleksander Pulver a, Evelyn Kiive b, Margus Kanarik ${ }^{\mathrm{c}}$ and Jaanus Harro a,c \\ a School of Natural Sciences and Health, Tallinn University, Narva Road 29, Astra \\ Building, 10120 Tallinn, Estonia \\ b Division of Special Education, Department of Education, University of Tartu, \\ Näituse 2, 50409 Tartu, Estonia \\ c Division of Neuropsychopharmacology, Department of Psychology, University \\ of Tartu, Ravila 14A Chemicum, 50411 Tartu, Estonia
}

\begin{abstract}
Orexins/hypocretins maintain wakefulness, increase appetite and participate in the coordination of stress response. We have recently provided evidence on the role of orexins in aggression, showing the association of the HCRTR1 genotype (rs2271933 G>A; leading to amino acid substitution Ile408Val) with aggressiveness or breach of law in four independent cohorts. Aggressive behaviour can be reward driven and hence we have examined the association of HCRTR1 rs2271933 genotype with different aspects of reward sensitivity in the birth cohort representative Estonian Children Personality Behaviour and Health Study. HCRTR1 genotype was associated with reward sensitivity in a gender dependent manner. Male HCRTR1 A/A homozygotes had higher Openness to Rewards and the overall reward sensitivity score while, in contrast, female A/A homozygotes scored lower than G-allele carriers in Openness to Rewards. In the
\end{abstract}


total sample, aggressiveness correlated positively with reward sensitivity, but this was on account of Insatiability by Reward. In contrast, the HCRTR1 A/A homozygotes had a positive association of aggressiveness and Openness to Rewards. Experience of stressful life events had a small but significant increasing effect on both aspects of reward sensitivity, and correlated in an anomalous way with reward sensitivity in the HCRTR1 A/A homozygotes. Conclusively, the higher aggressiveness of HCRTR1 A/A homozygotes appears based on a qualitative difference in sensitivity to rewards, in the form that suggests their lower ability to prevent responses to challenges being converted into overt aggression.

Keywords: orexins; HCRTR1; reward sensitivity; aggressiveness; gender

\section{Introduction}

Orexins or hypocretins are neuropeptides first described in 1998 (Sakurai et al., 1998; de Lecea et al., 1998) that are expressed in clusters of dorsomedial and lateral hypothalamus (Broberger et al., 1998) and involved in multiple physiological functions (Sutcliffe and de Lecea, 2000; Broberger and Hökfelt, 2001; Schwartz and Kilduff, 2015; Ferrario et al., 2016), including participation in the coordination of defence response (Johnson et al., 2012). Orexin release modulates the expression and extinction of fear memories (Flores et al., 2015) and the orexin neurons are activated in acute response to a variety of stressors; however, their role in chronic stress appears complex (Sargin, 2019), leading to the hypothesis that the role for orexins in neurotransmission is promoted during aversive conditions that elicit high arousal (Berridge et al., 2010). We have recently found evidence for a role of orexins in the other side of the flight/fight 
response, aggressiveness, by association of the HCRTR1 gene encoding the orexin OX1 receptor (Harro et al., 2019). The HCRTR1 gene variant (rs2271933, G1222A) in exon 7 that leads to amino acid substitution (Ile408Val) (Meerabux et al., 2005) was linked to aggressive behaviour or breach of law in four independent population samples by use of self-reports, interviews, and databases. In two population-representative birth cohort samples, male HCRTR1 rs2271933 A/A homozygotes were more aggressive than G-allele carriers. Female A/A homozygotes were also more aggressive if they had experienced higher number of adverse life events. The HCRTR1 genotype was also associated with re-occurrence of driving while impaired by alcohol and with involvement in traffic accidents (Harro et al., 2019). Another recent study has revealed that with each A-allele of the HCRTR1 rs2271933 there is less fMRI-measured activition in the inferior frontal cortex and more activation in the locus coeruleus (Gottschalk et al., 2019).

The only previous implication of orexins in human aggressiveness-related states had come from a unique study on epileptic patients that monitored release of orexin A in amygdala, and found an increase while subjects reported higher levels of anger (Blouin et al., 2013). Curiously, the highest increase of orexin levels was however related to experiencing positive emotions and during episodes of social interactions. Concluding from this and their previous animal studies showing activation of orexin neurons during positively but not during negatively motivated tasks (MacGregor et al., 2011), the authors suggested that orexin activity is necessary for positive emotion and motivation that drives social interaction. 
While motivation for positive social interaction does not pose direct health risks, simultaneous association of orexin release with anger brings about a possibility that the role of orexinergic neurotransmission in motivational activation can in occasion lead to less desirable behaviours. If resources are limited, aggression is a strategy to obtain rewards, and neurobiology of aggression and reward-related behaviour is closely intertwined (Panksepp, 1998; Aleyasin et al., 2018). Animal studies have strongly implicated orexins in reward processing (Harris et al., 2005; Moorman et al., 2017) and $\mathrm{OX}_{1}$ receptor antagonists are actively pursued as a potential avenue for treating drug addiction (Perrey and Zhang, 2020). It has been proposed that the role of orexins in reward seeking is in coordinating motivational activation when higher effort is required or external stimuli interfere with reward stimuli (James et al., 2017), and that $\mathrm{OX}_{1}$ receptor mediated signaling is an evolutionally conserved promoter of foraging (Barson, 2020). All this eveidence together suggests that orexins may be involved in the emergence of aggressive impulses in the context of reward seeking, possibly in particular in social contexts. Thus, we aimed at exploring whether the HCRTR1 rs2271933 genotype found to be associated with aggressiveness would also be associated with reward sensitivity. Reward sensitivity reflects the individually characteristic level of behavioural activation (Gray, 1994) and is a major component of temperament and personality reflecting the tendency to detect, pursue and derive pleasure from positive stimuli (Corr, 2009; Gray and McNaughton, 2000). It appears to emerge from neural networks with the mesotelencephalic dopaminergic circuitry at its core (Fu and Depue, 2019), and the latter receives a prominent input from the orexinergic neurons (Peyron et al., 
1998; Marcus et al., 2001). Because the higher aggressiveness in HCRTR1 rs2271933 A/A homozygotes had a different relationship with lifetime adversities in males $v s$ females, being independent of stressful life events in males, we hypothesized that reward sensitivity could contribute to the higher aggressiveness in HCRTR1 rs2271933 A/A males.

\section{Results}

2.1 HCRTR1 genotype and reward sensitivity in population-representative birth cohorts

Based on previous results on the association of the HCRTR1 rs2271933 genotype with aggressiveness, we had hypothetized that the relationship of the HCRTR1 rs2271933 genotype with reward sensitivity would be found in males but possibly not in females. Indeed, two-way ANOVA revealed statistically significant interaction effects for the Openness to Rewards scale and three of the four subscales, and several main effects of gender or genotype were also present. The HCRTR1 genotype was statistically significantly associated with three subscales of the Reward Openness and Insatiability Scale (ROIS): Excitement and Novelty, Social Experiences, and Giving in to Cravings. The interaction of the gender factor with genotype in case of Excitement and Novelty and the Openness to Rewards overall was largely owing to differences between male and female A/A homozygotes (Table 1; Fig 1): Male HCRTR1 A/A homozygotes had significantly higher Excitement and Novelty scores than G-allele carriers $[F(5,819)=4.10, p$ $=0.001$, partial $\left.\eta^{2}=0.02\right]$, but female HCRTR1 A/A homozygotes had lower scores than female G-allele carriers. The score of Social Experiences was generally 
higher in females than in males $\left[F(5,819)=2.60, p=0.024\right.$, partial $\left.\eta^{2}=0.02\right]$ but this was not the case for female HCRTR1 A/A homozygotes. Altogether this means that while in males each A-allele was associated with an increase in both subscales of Openness to Rewards, in females the HCRTR1 A/A-homozygotes had lower scores than female G-allele carriers, and the overall Openness to Rewards score was significantly higher in HCRTR1 A/A males but lower in HCRTR1 A/A females $\left[F(5,819)=2.47, p=0.031\right.$, partial $\left.\eta^{2}=0.02\right]$. Insatiability by Reward was not statistically significantly associated with the HCRTR1 genotype, but its Giving in to Cravings facet was significantly higher in HCRTR1 A/A males than male Gallele carriers $\left[F(5,819)=2.45, p=0.032\right.$, partial $\left.\eta^{2}=0.02\right]$; such a difference by genotype was not present in females. The Excessive Spending facet was neither statistically significantly associated with genotype nor with gender. 
Table 1. The Reward Openness and Insatiability Scale (ROIS) scores in males and females by HCRTR1 genotype (mean \pm SD).

\begin{tabular}{|c|c|c|c|c|c|c|}
\hline & $\begin{array}{l}\text { OR_Excitement } \\
\text { and Novelty }\end{array}$ & $\begin{array}{l}\text { OR_Social } \\
\text { experiences }\end{array}$ & $\begin{array}{l}\text { IR_Excessive } \\
\text { spending }\end{array}$ & $\begin{array}{l}\text { IR_Giving in to } \\
\text { cravings }\end{array}$ & $\begin{array}{l}\text { Openness to } \\
\text { Reward }\end{array}$ & $\begin{array}{l}\text { Insatiability by } \\
\text { Reward }\end{array}$ \\
\hline \multicolumn{7}{|l|}{ Males } \\
\hline $\mathrm{A} / \mathrm{A}$ & $\begin{array}{l}3.86 \pm 0.55^{* * *} \\
(\mathrm{n}=53)\end{array}$ & $\begin{array}{l}3.56 \pm 0.69 \\
(n=53)\end{array}$ & $2.45 \pm 0.73(n=52)$ & $\begin{array}{l}2.85 \pm 0.62 \\
(n=52)\end{array}$ & $\begin{array}{l}3.71 \pm 0.50^{*} \\
(\mathrm{n}=53)\end{array}$ & $2.65 \pm 0.58(n=52)$ \\
\hline $\mathrm{A} / \mathrm{G}$ & $\begin{array}{l}3.81 \pm 0.62 \\
(n=165)\end{array}$ & $\begin{array}{l}3.50 \pm 0.63^{* *} \\
(\mathrm{n}=165)\end{array}$ & $\begin{array}{l}2.42 \pm 0.80 \\
(n=163)\end{array}$ & $\begin{array}{l}2.56 \pm 0.73^{\# \#} \\
(n=163)\end{array}$ & $\begin{array}{l}3.65 \pm 0.53 \\
(n=165)\end{array}$ & $\begin{array}{l}2.49 \pm 0.68 \\
(n=163)\end{array}$ \\
\hline $\mathrm{G} / \mathrm{G}$ & $\begin{array}{l}3.63 \pm 0.63^{\#} \\
(n=122)\end{array}$ & $\begin{array}{l}3.46 \pm 0.69 * \\
(n=122)\end{array}$ & $\begin{array}{l}2.35 \pm 0.75 \\
(n=120)\end{array}$ & $\begin{array}{l}2.59 \pm 0.67 \# \\
(\mathrm{n}=120)\end{array}$ & $\begin{array}{l}3.54 \pm 0.55 \\
(n=122)\end{array}$ & $\begin{array}{l}2.47 \pm 0.61 \\
(n=120)\end{array}$ \\
\hline \multicolumn{7}{|c|}{ Females } \\
\hline $\mathrm{A} / \mathrm{A}$ & $3.46 \pm 0.64(\mathrm{n}=67)$ & $\begin{array}{l}3.53 \pm 0.65 \\
(\mathrm{n}=67)\end{array}$ & $2.62 \pm 0.90(\mathrm{n}=67)$ & $\begin{array}{l}2.72 \pm 0.70 \\
(\mathrm{n}=67)\end{array}$ & $\begin{array}{l}3.50 \pm 0.57 a \\
(n=67)\end{array}$ & $2.67 \pm 0.71(n=67)$ \\
\hline $\mathrm{A} / \mathrm{G}$ & $\begin{array}{l}3.73 \pm 0.67 \# \# \\
(\mathrm{n}=255)\end{array}$ & $\begin{array}{l}3.68 \pm 0.68 \\
(n=255)\end{array}$ & $\begin{array}{l}2.48 \pm 0.83 \\
(\mathrm{n}=254)\end{array}$ & $\begin{array}{l}2.59 \pm 0.65 \\
(n=254)\end{array}$ & $\begin{array}{l}3.71 \pm 0.58 \\
(n=255)\end{array}$ & $\begin{array}{l}2.53 \pm 0.62 \\
(n=254)\end{array}$ \\
\hline $\mathrm{G} / \mathrm{G}$ & $\begin{array}{l}3.65 \pm 0.66^{\#} \\
(\mathrm{n}=163)\end{array}$ & $\begin{array}{l}3.64 \pm 0.75 \\
(\mathrm{n}=163)\end{array}$ & $\begin{array}{l}2.47 \pm 0.89 \\
(n=162)\end{array}$ & $\begin{array}{l}2.73 \pm 0.74 \\
(\mathrm{n}=162)\end{array}$ & $\begin{array}{l}3.64 \pm 0.60 \\
(n=163)\end{array}$ & $\begin{array}{l}2.60 \pm 0.70 \\
(\mathrm{n}=162)\end{array}$ \\
\hline
\end{tabular}

OR - Openness to Reward; IR - Insatiability by Reward;

${ }^{\#} \mathrm{p}<0.05$; ${ }^{\#} \mathrm{p}<0.01$ different from the corresponding HCRTR1 A/A group; ap $<0.05$; a $\mathrm{p}<0.01$ different from the corresponding HCRTR1 A/G group;

${ }^{*} \mathrm{p}<0.05 ;{ }^{* *} \mathrm{p}<0.01 ;{ }^{* *} \mathrm{p}<0.001$ different from the corresponding female group.

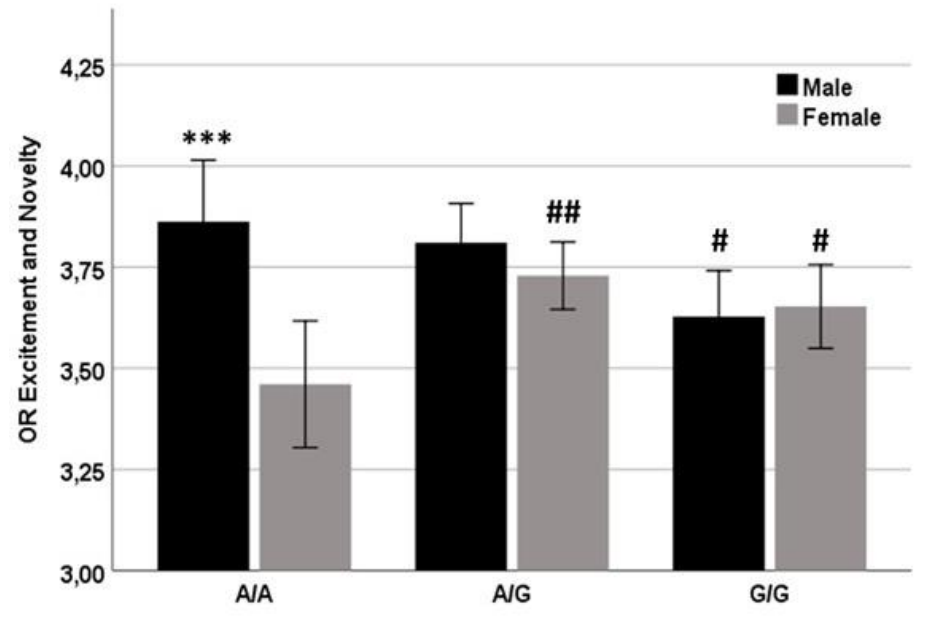

HCRTR1 rs2271933 genotype

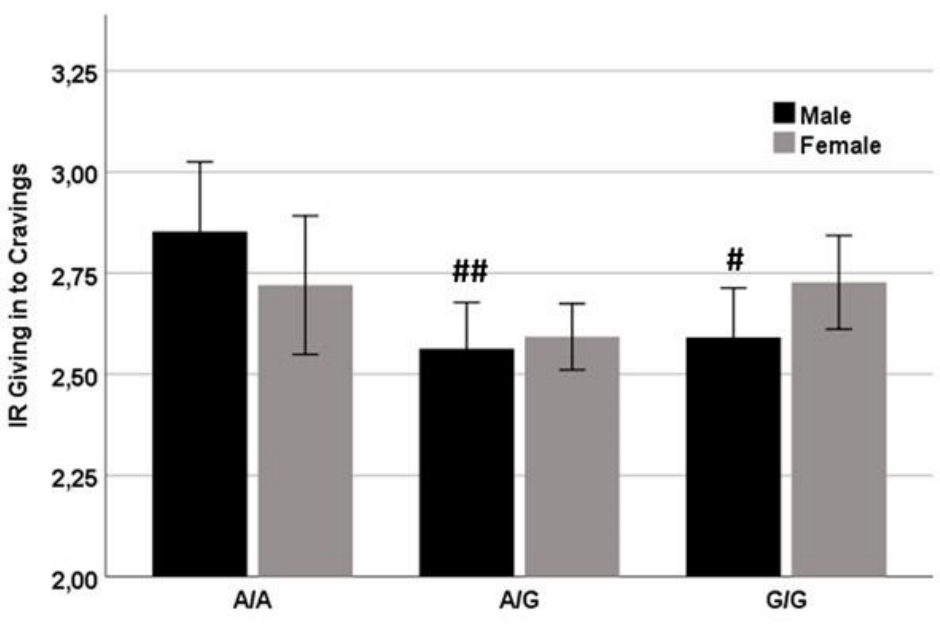

HCRTR1 rs2271933 genotype

A

B

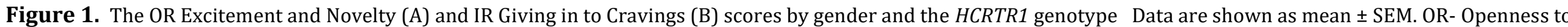

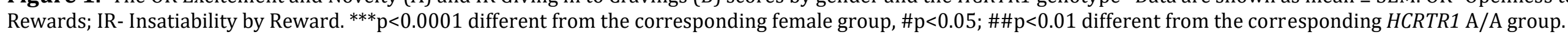




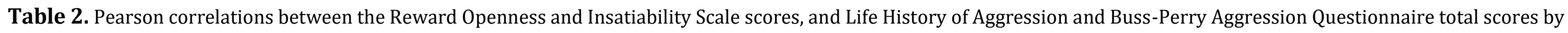
HCRTR1 genotype.

\begin{tabular}{|c|c|c|c|c|c|c|}
\hline & $\begin{array}{l}\text { OR_Excitement } \\
\text { and Novelty }\end{array}$ & $\begin{array}{l}\text { OR_Social } \\
\text { experiences }\end{array}$ & $\begin{array}{l}\text { IR_Excessive } \\
\text { spending }\end{array}$ & $\begin{array}{l}\text { IR_Giving in to } \\
\text { cravings }\end{array}$ & $\begin{array}{l}\text { Openness to } \\
\text { Reward }\end{array}$ & $\begin{array}{l}\text { Insatiability by } \\
\text { Reward }\end{array}$ \\
\hline \multicolumn{7}{|c|}{ A/A genotype $(\mathrm{n}=116)$} \\
\hline LHA Total & $0.24^{*}$ & 0.12 & $0.22^{*}$ & $0.25^{* *}$ & $0.21^{*}$ & $0.26^{* *}$ \\
\hline BPAQ Total & $0.20^{*}$ & 0.11 & $0.18^{*}$ & $0.29 * *$ & 0.18 & $0.27^{* *}$ \\
\hline \multicolumn{7}{|c|}{ A/G genotype $(\mathrm{n}=407)$} \\
\hline LHA Total & 0.07 & 0.02 & $0.23^{* * *}$ & $0.25^{* * *}$ & 0.05 & $0.27^{* * *}$ \\
\hline BPAQ Total & 0.05 & -0.04 & $0.33^{* * *}$ & $0.44^{* * *}$ & 0.01 & $0.44^{* * *}$ \\
\hline \multicolumn{7}{|c|}{$G / G$ genotype $(\mathrm{n}=278)$} \\
\hline LHA Total & 0.04 & 0.03 & $0.19 * *$ & $0.20 * *$ & 0.04 & $0.23^{* *}$ \\
\hline BPAQ Total & 0.01 & -0.02 & $0.41^{* * *}$ & $0.53^{* * *}$ & -0.02 & $0.54 * * *$ \\
\hline \multicolumn{7}{|c|}{ All groups $(\mathrm{n}=801)$} \\
\hline LHA Total & $0.08^{*}$ & 0.04 & $0.21 * * *$ & $0.24 * * *$ & 0.07 & $0.26 * * *$ \\
\hline BPAQ Total & 0.05 & -0.01 & $0.34^{* * *}$ & $0.46^{* * *}$ & 0.02 & $0.45^{* * *}$ \\
\hline
\end{tabular}

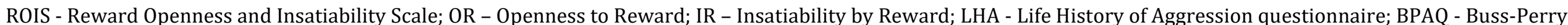
Aggression Questionnaire; ${ }^{*} \mathrm{p}<0.05 ; \mathrm{p}^{* *}<0.01 ; \mathrm{p}^{* * *}<0.0001$.

A

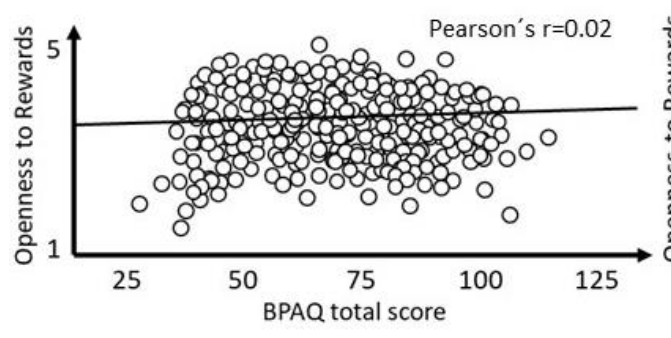

D

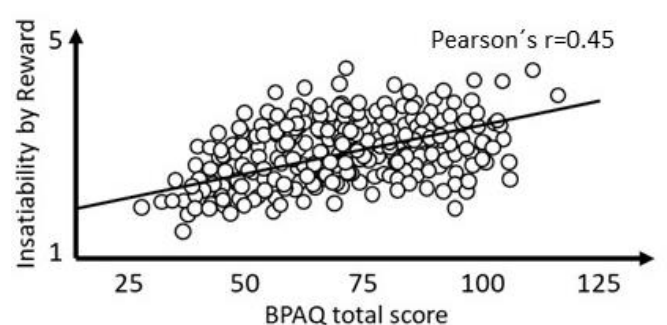

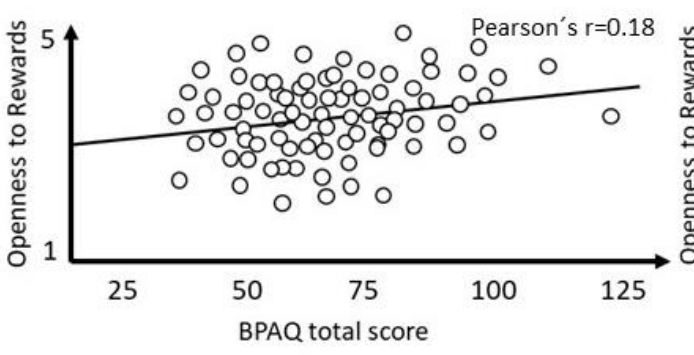

E

C
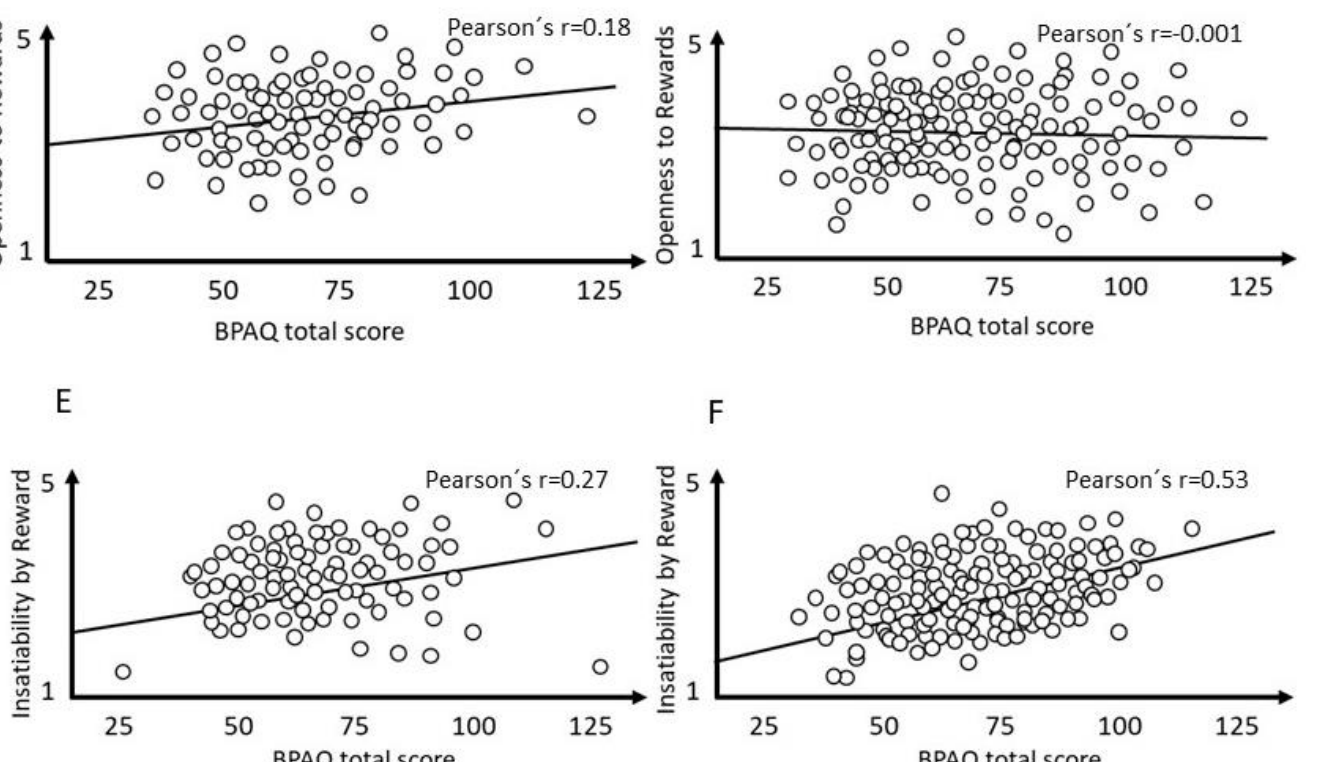

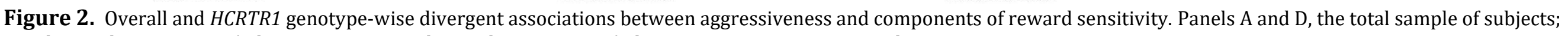
panels B and E, HCRTR1 A/A homozygotes; panels C and F, HCRTR1 G/G homozygotes. BPAQ- Buss and Perry Aggression Questionnaire. 
2.2 Relationship of aggressiveness and reward sensitivity, and its dependence on the HCRTR1 genotype

In the whole sample, Insatiability by Reward was positively correlated with scores of both Buss-Perry Aggression Questionnaire and the interview-based Life History of Aggression (Table 2). Openness to Reward had virtually no correlation with aggressiveness in the whole sample. We were particularly interested in the correlation between aggressiveness and reward sensitivity in the HCRTR1 A/A homozygotes, because this is the group previously found to present high aggressiveness. Intriguingly, the A/A-homozygotes had a weaker positive correlation between self-rated aggressiveness and Insatiability by Reward than the G-allele carriers (in A/A homozygotes the correlation between BPAQ total score and Insatiability by Reward was statistically significantly weaker than in A/G heterozygotes, $\mathrm{z}=1.84, \mathrm{p}=0.033$, and in $\mathrm{G} / \mathrm{G}$ homozygotes, $\mathrm{z}=2.93, \mathrm{p}=$ 0.002). In contrast, they had a positive correlation between aggressiveness and Openness to Rewards. Respective correlations in males and females were however largely similar (data not shown). An unexpected observation was made in comparison of correlations between reward sensitivity and the two measures of aggressiveness (Table 2; Fig. 2): In the HCRTR1 A/A homozygotes both selfrated and interview-based assessments correlated moderately with Insatiability by Reward and the correlations of the BPAQ total score and LHA total score with Insatiability by Reward were similar $(z=0.023, p=0.49)$. In contrast, correlation of the two measures of aggressiveness with this aspect of reward sensitivity was different in G-allele carriers so that each G-allele increased the correlation with self-assessed aggressiveness but not with interview-based assessment [correlations of the two measures of aggressiveness with Insatiability by Reward 
were statistically different $(\mathrm{z}=3.75, \mathrm{p}<0.0001$ and $\mathrm{z}=5.71, \mathrm{p}<0.0001$ for $\mathrm{A} / \mathrm{G}$ and $G / G$, respectively)].

\subsection{Reward sensitivity and stressful life experiences, relevance to aggressiveness}

Because we had previously found that in females the HCRTR1 genotype effect on aggressiveness depends on exposure to stressful life events (Harro et al., 2019), the interaction effect of the stressful life events (SLE), gender and HCRTR1 genotype on reward sensitivity was examined. However, no three-way interaction was found, but ANOVA revealed a statistically significant SLE main effects of stressful life events as well as the above described (2.1) interaction effect between genotype and gender on Openness to Reward. Both Openness to Rewards and Insatiability by Reward were slightly higher in the high SLE group as compared to the low SLE group $\left[F(1,754)=3.92, p=0.048\right.$, partial $\eta^{2}=0.05$ and $F(1,748)=3.92, p=0.048$, partial $\eta^{2}=0.05$; data not shown]. Neither did the covariance analysis reveal a role for stressful life events in the genotype by gender interaction. We also examined the correlation of the dimensional SLE measure with reward sensitivity. The SLE score was positively correlated with Insatiability by Reward, but the correlations were weak (Table 3). SLE did not correlate at all with Openness to Rewards in the whole sample, but in the HCRTR1 A/A-homozygotes a weak positive correlation between SLE and Openness to Rewards, in particular the Social Experiences subscale was revealed. 
Table 3

Pearson correlations between stressful life events and reward sensitivity measures by HCRTR 1 genotype.

\begin{tabular}{lllll}
\hline Reward sensitivity variable & SLE Total & SLE A/A & SLE A/G & SLE G/G \\
\hline Openness to Rewards & -0.02 & $0.22^{*}$ & -0.04 & -0.05 \\
OR Excitement and Novelty & -0.04 & 0.14 & -0.04 & -0.09 \\
OR Social Experiences & 0.01 & $0.23^{*}$ & -0.04 & -0.01 \\
Insatiability by Reward & $0.14^{* * *}$ & 0.12 & $0.15^{* *}$ & $0.13^{*}$ \\
IR Excessive Spending & $0.13^{* * *}$ & 0.14 & $0.15^{* *}$ & 0.10 \\
IR Giving in to Cravings & $0.10^{* *}$ & 0.05 & 0.09 & 0.12 \\
\hline
\end{tabular}

SLE stressful life event scores; ${ }^{*} \mathrm{p}<0.05,{ }^{* *} \mathrm{p}<0.01,{ }^{* * *} \mathrm{p}<0.001$

\section{Discussion}

We have found that the HCRTR1 rs2271933 (G1222A) genotype that causes an amino acid substitution (Ile408Val) of the $\mathrm{OX}_{1}$ receptor protein in a region that is suggested to interact with G proteins or other proteins (Thompson et al., 2014) and has been associated with aggressiveness or accident-prone law-breaching behaviour in multiple cohorts (Harro et al., 2019) is also associated with reward sensitivity. Aggressiveness belongs to the behavioural repertoire of coordinated adaptive response to environmental challenges (van Kampen, 2015) and in this response different nuclei of the hypothalamus play a critical role (Kruk, 2014). Reward sensitivity can shape the emergence of aggressive behaviour through distinct pathways and, interestingly, the association of aspects of reward sensitivity and aggressive behaviour was HCRTR1 rs2271933 genotype dependent.

In a previous analysis (Pulver et al., 2020) we dissected reward sensitivity into two major components with hardly any co-variance, one representing striving 
toward reward multiplicity and another describing the fixation to a given reward. We found that only the latter, Insatiability by Reward, was associated with symptoms of ADHD and the TPH2 -703G/T polymorphism; also, only this component of reward sensitivity was associated with the ANGER factor of the Affective Neuroscience Personality Scale (Davis and Panksepp, 2011). Subsequently, we have found that Insatiability by Reward, but not Openness to Rewards, is associated with the increase of body mass index and other measures of obesity from childhood to young adulthood (Katus et al., 2020). Thus, the two aspects of reward sensitivity have substantially different consequences to behaviour. Herewith we have extended these findings to demonstrate that the trait of being strongly fixated on a particular reward is positively correlated to both self-assessed and interview-based aggressiveness, while striving toward multiple rewards, generally, is not.

A role for orexins in reward seeking has been proposed in a number of experimental studies (Tyree et al., 2018), but the results have not been uniform. In order to explain the apparent controversies, orexins have been suggested to coordinate motivational activation in effortful conditions (James et al., 2017). This would be in line with the notion that neuropeptides gain significance at higher neural activity (Hökfelt et al., 2000; Hökfelt et al., 2018). Neuroanatomical and functional circuitry studies have indeed suggested that the orexin system modulates reward processing through neural substrates that are crucial in the control of arousal ( $\mathrm{Li}$ and de Lecea, 2020). For example, the locus coeruleus received the densest extrahypothalamic projections from the orexin neurons (Peyron et al., 1998), and predominantly express the $\mathrm{OX}_{1}$ receptors. Of note, the 
HCRTR1 rs2271933 genotype was recently found to be associated with activation of the locus coeruleus, this being higher with the A-allele in an additive manner (Gottschalk et al., 2019).

No significant gender differences have emerged in the structure of reward sensitivity or the relationship of its components with a number of personality and behavioural measures. But a large body of evidence suggests the existence of relevant gender differences in personality and behaviour. For example, men in general display higher levels of narcissism, Machiavellianism, and psychopathy (Muris et al., 2017), impulsivity (Cross et al., 2011), and are physically more aggressive than women (Björkqvist, 2018). Important gender differences in dopaminergic mechanisms mediating reward exist (Becker and Chartoff, 2019) and the present study suggests that the orexin system may contribute to the differences in the development of male and female aggressiveness through neurobiological underpinnings of reward sensitivity. Orexin neurons in the lateral hypothalamus are in bidirectional cross-talk with dopamine neurons in the ventral tegmental area (Liu et al., 2020) and dopamine can elicit opposite behavioural effects via input to orexin neurons through distinct receptor subtypes (Linehan et al., 2019). Indeed significant sex differences in the orexin system have been described, with higher orexin system activity in females and sex-specific interactions with the HPA axis (Grafe and Bhatnagar, 2020). Studies using pharmacological $\mathrm{OX}_{1}$ receptor blockade have demonstrated that while in both male and female animals sucrose self-administration in condition of food restriction involves orexin signaling, only in males the $\mathrm{OX}_{1}$ receptor mediated 
signal is involved in cue-reinstated sucrose seeking (Cason and Aston-Jones, 2014).

Aggressive behaviour had been found to relate to the HCRTR1 rs2271933 genotype irrespective of life events in males but dependent on higher exposure to adverse events in females (Harro et al., 2019). We hypothesized that male HCRTR1 rs2271933 A/A homozygotes, with markedly higher aggressiveness, are driven by higher Insatiability by Reward. Indeed we did find significantly higher scores in one of the two sub-scales of Insatiability by Reward, and a tendency toward higher overall score of this measure in male HCRTR1 rs2271933 A/A homozygotes. We did, however, also observe in males higher scores by each Aallele in the other aspect of reward sensitivity, Openness to Rewards. Moreover, the most evident genotype effect was the difference between male and female HCRTR1 rs2271933 A/A homozygotes with regard to Openness to Rewards and, in particular, its sub-scale Excitement and Novelty, with male A/A homozygotes having the highest but female A/A homozygotes the lowest scores. In the Social Experiences subscale the male A/A homozygotes did not disply any difference from other genotypes: While the behaviour of A/A males has been found as more frequently anti-social, this would refer to single acts of violationg social norms (Harro et al., 2019) while the subscale measures motivation of social exchange using items such as 'enjoys big parties' or 'is modest in socializing' (reversed item; Pulver et al., 2020).

Because the association of the Openness to Rewards with aggressiveness was not expected, we examined the correlation between aggressiveness and the 
components of reward sensitivity by the HCRTR1 rs2271933 genotype. Overall, both self-reported and interview-based aggressiveness measures were in positive correlation with Insatiability by Reward, but not with Openness to Rewards. Nevertheless, these relationships were different in the HCRTR1 rs2271933 A/A homozygotes (who form 15\% of the total sample): Only in this genotype the aggressiveness measures were related to Openness to Rewards, whereas correlation of Insatiability by Reward with the self-rated aggressiveness measure was lower than in other genotypes. Different measures of aggressiveness are in significant but only moderate correlation (Kiive et al., 2017), and the major difference in the measures obtained by the BPAQ and LHA interview, besides one being self-administered and another interview-based, is in the fact that the former includes feelings and thoughts while the latter is devoted to aggressive behaviour that has actually occurred, and quantitates its frequency. This difference is also reflected in the highest correlation of the LHA score with the Physical Aggression component of the BPAQ (Kiive et al., 2017).

Direct evidence at the biochemical level regarding functionality of HCRTR1 rs2271933 (Ile408Val) variation is not available, but it has been suggested that the valine in the respective position could promote the phosphorylation of the immediately adjacent serine and alter signal transduction upon activation of the orexin OX 1 receptor (Meerabux et al., 2005). The HCRTR1 rs2271933 genotype has previously been associated with migraine (Rainero et al., 2011b; Kowalska et al., 2018) and mood disorders (Rainero et al., 2011a), the ANGER factor of the Affective Neuroscience Personality Scale, and with aggressiveness (Harro et al., 2019). Interestingly, the A-allele of the HCRTR1 rs2271933 genotype was also 
associated with higher proneness to traffic accidents according to police registry and traffic insurance database, and the A/A homozygotes had the risk of recidive of drunk driving (Harro et al., 2019). The latter finding may offer insight into the role of this genetic variation and of the $\mathrm{OX}_{1}$ receptor in regulation of behaviour, as in this longitudinal study the HCRTR1 rs2271933 A/A homozygocity was associated with drunk driving only in the group with previous drunk driving record but not in control subjects or in drivers with speed limit violations. Irresponsible use of alcohol thus appears as a specific risk factor for the HCRTR1 rs2271933 A/A homozygotes, and is known to strongly correlate with manifest aggressive behaviour. In the stop-signal reaction time task, administration of a OX $\mathrm{X}_{1}$-receptor antagonist reduced motivation to perform the task but did not affect inhibitory control (Wiskerke et al., 2019). This finding suggests that $\mathrm{OX}_{1}$ receptors are not contributing to aggression control through simple real-time inhibition, but intoxication by alcohol could change this. As a working hypothesis, it should be further examined whether the distinct relationship of aggressiveness with reward sensitivity in HCRTR1 rs2271933 A/A homozygotes is derived from misinterpretations and failed inhibitions while engaging in novel and exciting situations instead of the more common ground of aggressiveness in the inflexible fixation on specific rewards.

Higher exposure to adversity during childhood and adolescence increased reward sensitivity but the effect was minor and could not explain the genotype by gender interactions. Interestingly, the matrix of weak but statistically significant correlations again suggested a dissociation between HCRTR1 rs2271933 A/A homozygotes and G-allele carriers, as only in the former the 
exposure to stressful events was (positively) related to Openness to Rewards, in particular to Social Experiences in young adulthood. Limited sample size did not permit a detailed mediation analysis, but speculatively a hypothesis could be put forward that in the HCRTR1 rs2271933 A/A homozygotes life stress increases strive toward social acceptance, that could increase the risk of occasionally resulting in transformation into inappropriate aggressive behaviour.

Increase in orexin A levels in amygdala was found during anger (Blouin et al., 2013) but even higher release of orexin was observed during experiencing positive emotions and in episodes of social interaction. The two aspects of reward sensitivity are differently related to not only to anger but all basic emotions: Of the facets of the Affective Neuroscience Personality Scale, Insatiability by Reward scores co-varied with ANGER, SADNESS and FEAR, but Openness to Rewards with SEEKING, PLAY and CARE (Pulver et al., 2020). In animal experiments the orexin neurons were found to fire during positively but not during negatively motivated tasks (MacGregor et al., 2011). Thus, previous studies and the present analysis converge to suggest, albeit indirectly, that the involvement of orexin in aggressive and antisocial behaviour is mediated by inappropriate interpretation of social cues. Proper function of orexin neurons is crucial to appropriately timed transitions between arousal states, and for taking context-specific actions (Burdakov, 2020). The major genotype by gender interaction with regard to openness to rewards, especially excitement and novelty, may help to explain the difference between expression of aggressiveness in male and female HCRTR1 rs2271933 A/A homozygotes. Higher striving towards exciting and novel situations can expose the male A/A homozygotes to 
situations that provoke aggression. In females, this risk appears as being compensated for if not exposed to adversities. Nevertheless, this study is limited in collecting relevant data in young adulthood and thus any hypotheses regarding developmental strategies remain speculative; also the sample size does not support a detailed analysis given the prevalence of the genotype of particular interest. Another limitation concerns the novel reward sensitivity instrument that was developed post hoc using a large item pool in a Fennic language, and requires further characterization together with related instruments. The strength of the study however includes the exceptional representation of eligible population-based cohorts and uniform data collection procedure in a laboratory setting.

Conclusively, male HCRTR1 rs2271933 A/A homozygotes, previously found to display higher aggressiveness, reported higher striving towards multiple rewards, whereas A/A females, previously found to show higher aggressiveness only in association with severe life events, were less open to excitement and novelty. These findings support the role of orexins in reward sensitivity and the notion of different pathways to aggressiveness in males and females.

\section{Method}

\subsection{Sample}

This study was carried out on the Estonian sample of the European Youth Heart Study (1998/1999), which was subsequently incorporated into the longitudinal Estonian Children Personality Behaviour and Health Study (ECPBHS). The rationale and procedure of sample formation, and further data collection waves 
have been described elsewhere in detail (Harro et al., 2001; Harro et al., 2019). ECPBHS is highly representative of two birth cohorts of a local population, as $79 \%$ of subjects of the randomized regional sample participated in the original data collection. All the subjects are of European descent. Data collection for the presented analyses was conducted at ages 25 (both cohorts) and 33 (only the older cohort). The original size of the total sample is $n=1238$, but all data necessary for the analyses presented in this paper was $n=818$ to 825 . This study was carried out in accordance with the code of ethics as per Declaration of Helsinki, it was approved by the Ethics Review Committee on Human Research of the University of Tartu, and written informed consent was obtained from all the participants.

\subsection{Assessment of reward sensitivity}

In order to take into account the inner structure of reward sensitivity, the Reward Openness and Insatiability Scale (ROIS) was used to assess reward sensitivity (Pulver et al., 2020). In brief, ROIS comprises 28 items equally divided between two higher-order factors, Openness to Rewards and Insatiability by Reward, that describe striving toward multiplicity of rewards and excessive fixation to particular reward, respectively. Openness to Rewards includes two facets, Excitement and Novelty, and Social Experiences. Insatiability by Reward comprises Excessive Spending and Giving in to Cravings. While items belonging to the two factors were the product of an exploratory principal component analysis, the factors do not correlate significantly with each other, whereas the facets that form the factors correlate with each other moderately ( $r=0.45$ and 
0.48). ROIS scores were available for 818 or 825 participants for the two subscales.

\subsection{Measures of aggressiveness}

\subsubsection{Self-report questionnaire}

The 29-item Buss-Perry Aggression Questionnaire (BPAQ; Buss and Perry, 1992) assesses four aspects of aggressive behaviour: Physical aggression, Verbal aggression, Anger, and Hostility. Participants rated each statement on a 5-point Likert Scale (uncharacteristic $=1$, characteristic=5). Data of Buss-Perry Aggression Questionnaire were available for 926 subjects.

\subsubsection{Aggressiveness assessment by interview}

The Life History of Aggression interview (LHA; Coccaro et al., 1997) was carried out by an experienced clinical psychologist. Items were scored only for the history of actual behavior. LHA has three subscales: Aggression (temper tantrums, verbal aggression, indirect aggression, non-specific fighting, and physical assault against people); Consequences/Antisocial Behaviour (school disciplinary problems, problems with supervisors, antisocial behaviour not resulting in police involvement, and antisocial behaviour involving the police); and Self-Directed Aggression (self-injurious behavior, and suicide attempts). Each item was rated on a 5-point scale, ranging from $0=$ No events to $5=$ More events than can be counted. Data of LHA were available for 922 subjects. 


\subsection{Assessment of stressful life experiences}

Stress experienced in childhood was measured at age 15 with a list of 21 adverse life experiences reported by participants or their parents, which included questions about parental death and divorce/separation, absence of both parents, unemployment in the family, financial problems and poverty in the household, poor living conditions, poor health and chronic diseases, serious illness of a family member, death of a close relative, trauma, fear of school, bullying at school and humiliation at home (Laas et al., 2014). The events were recorded as dichotomous variables (present or not present) and were then counted to form the number of experienced stressful life events (SLE). For group-wise comparison, two categories of the SLE (Low SLE, 0-2 events and High SLE, $>2$ events) were constructed on the basis of median value of SLE score $(M d=2)$. Data were available for 557 subjects in the ECPBHS younger cohort and for 594 subjects in the older cohort.

\subsection{Genotyping HCRTR1 rs2271933}

This was carried out as previously described (Harro et al., 2019). Genomic DNA was extracted from venous blood samples using Qiagen QIAamp® DNA Blood Midi Kit. The real-time polymerase chain reaction (RT-PCR) for genotyping the HCRTR1 rs2271933 polymorphism was performed using a TaqMan Pre-Designed SNP Genotyping Assay (Applied Biosystems; Foster City, CA, USA) C_15961465_10 containing primers and fluorescent probes. Genotyping reactions were performed in a total volume of $10 \mu \mathrm{l}$ with $\sim 25 \mathrm{ng}$ of template 
DNA. RT-PCR reaction components and final concentrations were as follows: 1:5 5 x HOT FIREPol ${ }^{\circledR}$ Probe qPCR Mix Plus (ROX) (Solis BioDyne) and 1:20 $80 \mathrm{x}$ TaqMan Primers Probe. Context sequence [VIC/FAM] was as follows: CTTGTCCTTGCAGAGCCGATGCTCC[A/G]TCTCCAAAATCTCTGAGCATGTGGT. Reactions were performed on the Applied Biosystems ViiA ${ }^{\mathrm{TM}} 7$ Real-Time PCR System. The amplification procedure consisted of an initial denaturation step at $95^{\circ} \mathrm{C}$ for $12 \mathrm{~min}$ and 40 cycles of $95^{\circ} \mathrm{C}$ for $15 \mathrm{~s}$ and $60^{\circ} \mathrm{C}$ for $1 \mathrm{~min}$. Positive and negative controls were added to each reaction plate. No inconsistencies occurred. Genotyping was performed blind to all phenotypic data. Allele frequencies agreed with National Center for Biotechnology Information database (minor allele frequency around 0.4;

https://www.ncbi.nlm.nih.gov/snp/rs2271933\#frequency tab) and published reports. All the subjects were of European origin, and genotype frequencies of all samples were in Hardy-Weinberg equilibrium.

\subsection{Statistical analysis}

Statistical analysis was carried out using the SPSS v. 25 software. Two-way between-groups analyses of variance (ANOVA) were performed to investigate gender and genotype interactions in reward sensitivity. The dependent variables included the Reward Openness and Insatiability Scale (ROIS) summary score, the Openness to Reward (OR) scale and its sub-scales Excitement and Novelty subscale and Social experiences, and the Insatiability by Reward (IR) scale and its sub-scales Excessive spending and Giving in to cravings.

The independent variables (fixed factors) were the HCRTR1 rs2271933 genotype (A/A, A/G, G/G) and gender. A three-way between-groups univariate analysis of 
variance (ANOVA) was also performed to investigate the stressful life experience, gender and genotype interactions in reward sensitivity. Two-way analysis of covariance (ANCOVA) was used to examine the possible covariate effect of stressful life events on the association between genotype, gender and reward sensitivity. Preliminary assumption testing was conducted to check for normality, outliers, homogeneity of variance, with no serious violations noted. In case of significant ANOVA interaction effects the differences between means of the 6 groups (two gender levels by three genotype levels) was investigated by Fisher LSD post hoc test. Associations between the test scores were assessed by Pearson correlation. The z-statistic was used for comparison of correlations. Analysis was performed and data are presented as the mean item score (i.e., sum of the items is divided by number of items in scale). In the statistical analysis, the conventional 5\% level was used to assess the significance.

\section{Acknowledgements}

We are grateful to the ECPBHS study participants, their parents and the whole ECPBHS Team. This work was supported by Estonian Research Council Project IUT20-40, the EC Horizon 2020 project CoCA (H2020-PHC-2015-667302), and the Tallinn University ASTRA project TU TEE financed by the European Union European Regional Development Fund (2014-2020.4.01.16-0033).

Declarations of Interest: None.

\section{References}


Aleyasin, H., Flanigan, M.E., Russo, S.J., 2018. Neurocircuitry of aggression and aggression seeking behavior: Nose poking into brain circuitry controlling aggression. Curr. Opin. Neurobiol. 49, 184-191.

Barson, J.R., 2020. Orexin/hypocretin and dysregulated eating: promotion of foraging behavior. Brain Res. 1731, 145915.

Becker J.B., Chartoff E., 2019. Sex differences in neural mechanisms mediating reward and addiction. Neuropsychopharmacology 44, 166-183. doi: 10.1038/s41386-018-0125-6.

Berridge, C.W., Espana, R.A., Vittoz, N.M., 2010. Hypocretin/orexin in arousal and stress. Brain Res. 1314, 91-102.

Blouin, A.M., Fried, I., Wilson, C.L., Staba, R.J., Behnke, E.J., Lam, H.A., Maidment, N.T., Karlsson, K.A., Lapierre, J.L., Siegel, J.M., 2013. Human hyprocretin and melanin-concentrating hormone levels are linked to emotion and social interaction. Nature Comm. 4, 1547.

Broberger, C., De Lecea, L., Sutcliffe, J.G., Hökfelt, T., 1998. Hypocretin/orexinand melanin-concentrating hormone-expressing cells form distinct populations in the rodent lateral hypothalamus: Relationshipto the neuropeptide $\mathrm{Y}$ and agouti gene-related protein systems. J. Comp. Neurol. 402, 460-474.

Broberger, C., Hökfelt, T., 2001. Hypothalamic and vagal neuropeptide circuitries regulating food intake. Physiol. Behav. 74, 669-682.

Buss, A.H., Perry, M., 1992. The aggression questionnaire. J. Pers. Soc. Psychol. 63, 452-259.

Cason, A.M., Aston-Jones, G., 2014. Role of orexin/hypocretin in conditioned sucrose-seeking in female rats. Neuropharmacology 86, 97-102.

Coccaro, E.F., Berman, M.E., Kavoussi, R.J., 1997. Assessment of life history of aggression: development and psychometric characteristics. Psychiatry Res. 73, 147-157.

Corr, P.J., 2009. The reinforcement sensitivity theory of personality, in: Corr, P. J., Matthews, G. (Eds.), The Cambridge Handbook of Personality Psychology. Cambridge University Press, Cambridge, England, pp. 347-376.

Björkqvist, K., 2018. Gender differences in aggression. Curr. Opin. Psychol., 19, 39-42. doi: 10.1016/j.copsyc.2017.03.030. 
Burdakov, D., 2020. How orexin signals bias action: Hypothalamic and accumbal circuits. Brain Res. 1731, 145943.

Cross, C.P., Copping, L.T., Campbell, A., 2011. Sex differences in impulsivity: a meta-analysis. Psychol. Bull., 137, 97-130. doi: 10.1037/a0021591.

Davis, K.L., Panksepp, J., 2011. The brain's foundations of human personality and the Affective Neuroscience Personality Scales. Neurosci. Biobehav. Rev. 35, 1946-1958.

de Lecea, L., Kilduff, T.S., Peyron, C., Gao, X., Foye, P.E., Danielson, P.E., Fukuhara, C., Battenberg, E.L., Gautvik, V.T., Bartlett, F.S. 2nd, Frankel, W.N., van den Pol, A.N., Bloom, F.E., Gautvik, V.T., Sutcliffe, J.G., 1998. The hypocretins: hypothalamus-specific peptides with neuroexcitatory activity. Proc. Natl. Acad. Sci. U.S.A. 95, 322-327.

Ferrario, C.R., Labouebe, G., Liu, S., Nieh, E.H., Routh, V.H., Xu, S., O'Connor, E.C., 2016. Homeostasis meets motivation in the battle to control food intake. J. Neurosci. 36, 11469-11481.

Flores, A., Saravia, R., Maldonado, R., Berrendero, F., 2015. Orexins and fear: implications for the treatment of anxiety disorders. Trends Neurosci. 38, $550-559$.

Fu, Y., Depue, R.A., 2019. A novel neurobiological framework of the effects of positive early postnatal experience on incentive and consummatory reward sensitivity. Neurosci. Biobehav. Rev. 107, 615-640.

Gottschalk, M.G., Richter, J., Ziegler, C., Schiele, M.A., Mann, J., Geiger, M.J., Schartner, C., Homola, G.A., Alpers, G.W., Büchel, C., Fehm. L., Fydrich, C., Gerlach, A.L., Gloster, A.T., Helbig-Lang, S., Kalisch, R., Kircher, T., Lang, T., Lonsdorf, T.B., Pané-Farré, C.A., Ströhle, A., Weber, H., Zwanzger, P., Arolt, V., Romanos, M., Wittchen, H.U., Hamm, A., Pauli, P., Reif, A., Deckert, J., Neufang, S., Höfler, F., Domschke, K., 2019. Orexin in the anxiety spectrum: Association of a HCRTR1 polymorphism with panic disorder/agoraphobia, CBT treatment response and fear-related intermediate phenotypes. Transl. Psychiatry 9, 75. doi: 10.1038/s41398-019-0415-8.

Grafe, L.A., Bhatnagar, S., 2020. The contribution of orexins to sex diferences in the stress response. Brain Res. 1731, 145893. 
Gray, J.A., 1994. Three fundamental emotion systems, in: Ekman, P., Davidson, J.R. (Eds.), The Nature of Emotion: Fundamental Questions. Oxford University Press, New York, pp. 243-247.

Gray, J.A., McNaughton, N., 2000. The Neuropsychology of Anxiety: An Enquiry into the Functions of the Septo-Hippocampal System, second edition. Oxford University Press, Oxford, England.

Harris, G.C., Wimmer, M., Aston-Jones, G., 2005. A role for lateral hypothalamic orexin neurons in reward seeking. Nature 437, 556-559.

Harro, J., Laas, K., Eensoo, D., Kurrikoff, T., Sakala, K., Vaht, M., Parik, J., Mäestu, J., Veidebaum, T., 2019. Orexin/hypocretin receptor gene (HCRTR1) variation is associated with aggressive behaviour. Neuropharmacology 156, 107527. doi: 10.1016/j.neuropharm.2019.02.009.

Harro, M., Eensoo, D., Kiive, E., Merenäkk, L., Alep, J., Oreland, L., Harro, J., 2001. Platelet monoamine oxidase in healthy 9- and 15-years old children: the effect of gender, smoking and puberty. Prog. Neuropsychopharmacol. Biol. Psychiatry 25, 1497-1511.

Hökfelt, T., Barde, S., Xu, Z.D., Kuteeva, E., Rüegg, J., Le Maitre, E., Risling, M., Kehr, J., Ihnatko, R., Theodorsson, E., Palkovits, M., Deakin, W., Bagdy, G., Juhasz, G., Prud'homme, H.J., Mechawar, N., Diaz-Heijtz, R., Ögren, S.O., 2018. Neuropeptide and small transmitter coexistence: Fundamental studies and relevance to mental illness. Front. Neural Circuits. 12, 106. doi: 10.3389/fncir.2018.00106.

Hökfelt, T., Broberger, C., Xu, Z.Q., Sergeyev, V., Ubink, R., Diez, M., 2000. Neuropeptides--an overview. Neuropharmacology 39, 1337-1356.

James, M.H., Mahler, S.V., Moorman, D.E., Aston-Jones, G., 2017. A decade of orexin/hypocretin and addiction: Where are we now? Curr. Top. Behav. Neurosci. 33, 247-281.

Johnson, P.L., Molosh, A., Fitz, S.D., Truitt, W.A., Shekhar, A., 2012. Orexin, stress, and anxiety/panic states. Prog. Brain Res. 198, 133-161.

Katus, U., Villa, I., Ringmets, I., Pulver, A., Veidebaum, T., Harro, J., 2020. The role of reward sensitivity in obesity and its association with Transcription Factor AP-2B: A longitudinal birth cohort study. Neurosci. Lett. 735, 135158. 
Kiive, E., Laas, K., Vaht, M., Veidebaum, T., Harro, J., 2017. Stressful life events increase aggression and alcohol use in young carriers of the GABRA2 rs279826/rs279858 A-allele. Eur. Neuropsychopharmacol. 27, 816-827.

Kowalska, M., Kapelusiak-Pielok, M., Grzelak, T., Wypasek, E., Kozubski, W., Dorszewska, J., 2018. The new *G29A and G1222A of HCRTR1, 5-HTTLPR of SLC6A4 polymorphisms and hypocretin-1, serotonin concentrations in migraine patients. Front. Mol. Neurosci. 11, 191. DOI: 10.3389/fnmol.2018.00191.

Kruk, M., 2014. Hypothalamic attack: A wonderful artifact or a useful perspective on escalation and pathology in aggression? A viewpoint. Curr. Top. Behav. Neurosci. 17, 143-188.

Laas, K., Reif, A., Kiive, E., Domschke, K. Lesch, K.P. Veidebaum, T. Harro, J. , 2014. A functional NPSR1 gene variant and environment shape personality and impulsive action: a longitudinal study. J. Psychopharmacol. 28, 227-236. doi:10.1177/0269881112472562.

Li, S.-H., de Lecea, L., 2020. The hypocretin (orexin) system: From a neural circuitry perspective. Neuropharmacology 167, 107993.

Linehan, V., Rowe, T.M., Hirasawa, M., 2019. Dopamine modulates excitatory transmission to orexin neurons in a receptor subtype-specific manner. Am. J. Physiol. Regul. Integr. Comp. Physiol. 316, R68-R75.

Liu, J.J., Mirabella, V.R., Pang, Z.P., 2020. Cell type- and pathway-specific synaptic regulation of orexin neurocircuitry. Brain Res. 1731, 145974.

Marcus, J.N., Aschkenasi C.J., Lee, C.E., Chemelli, R.M., Saper, C.B., Yanagisawa, M., Elmquist, J.K., 2001. Differential expression of orexin receptors 1 and 2 in the rat brain. J. Comp. Neurol. 435, 6-25.

McGregor, R., Wu, M. -F., Barber, G., Ramanathan, L. \& Siegel, J. M., 2011. Highly specific role of hypocretin (orexin) neurons: differential activation as a function of diurnal phase, operant reinforcement versus operant avoidance and light level. J. Neurosci. 31, 15455-15467.

Meerabux, J., Iwayama, Y., Sakurai, T., Ohba, H., Toyota, T., Yamada, K., Nagata, R., Irukayama-Tomobe, Y., Shimizu, H., Yoshitsugu, K., Ohta, K., Yoshikawa, T., 2005. Association of an orexin 1 receptor $408^{\mathrm{Val}}$ variant with polydipsiahyponatremia in schizophrenic subjects. Biol. Psychiat. 58, 401-407. 
Moorman, D.E., James, M.H., Kilroy, E.A., Aston-Jones, G., 2017.

Orexin/hypocretin-1 receptor antagonism reduces ethanol selfadministration and reinstatement selectively in highly-motivated rats. Brain Res. 1654, 34-42.

Muris, P., Merckelbach, H., Otgaar, H., Meijer, E., 2017. The malevolent side of human nature: A meta-analysis and critical review of the literature on the Dark Triad (narcissism, machiavellianism, and psychopathy). Perspect. Psychol. Sci. 2, 183-204. doi: 10.1177/1745691616666070.

Panksepp, J., 1998. Affective Neuroscience: The Foundations of Human and Animal Emotions, Oxford University Press.

Perrey, D.A., Zhang, Y., 2020. Therapeutics development for addiction: Orexin-1 receptor antagonists. Brain Res. 1731, 145922.

Peyron, C., Tighe, D.K., van den Pol, A.N., de Lecea, L., Heller, H.C., Sutcliffe, J.G., Kilduff, T.S., 1998. Neurons containing hypocretin (orexin) project to multiple neuronal systems. J. Neurosci. 18, 9996-1015.

Pulver, A., Kiive, E., Harro, J., 2020. Reward sensitivity, Affective Neuroscience personality, symptoms of attention deficit/hyperactivity disorder, and TPH2 -703G/T (rs4570625) genotype. Acta Neuropsychiat. (in press).

Rainero, I., Ostacoli, L., Rubino, E., Gallone, S., Picci, L.R., Fenoglio, P., Negro, E., Rosso, C., De Martino, P., De Marchi, M., Furlan, P.M., Pinessi, L., 2011 a. Association between major mood disorders and the hypocretin receptor 1 gene. J. Affect. Disord. 130, 487-491.

Rainero, I., Rubino, E., Gallone, S., Fenoglio, P., Picci, L.R., Giobbe, L., Ostacoli, L., Pinessi, L., 2011b. Evidence for an association between migraine and the hypocretin receptor 1 gene. J. Headache Pain 12, 193-199.

Sakurai, T., Amemiya, A., Ishii, M., Matsuzaki, I., Chemelli, R.M., Tanaka, H., Williams, S.C., Richardson, J.A., Kozlovski, G.P., Wilson, S., Arch, J.R., Buckingham, R.E., Haynes, A.C., Carr, S.A., Annan, R.S., McNulty, D.E., Liu, W.S., Terrett, J.A., Elshourbagy, N.A., Bergsma, D.J., Yanagisawa, M., 1998. Orexins and orexin receptors: a family of hypothalamic neuropeptides and G proteincoupled receptors that regulate feeding behavior. Cell 92, 573-585.

Sargin, D., 2019. The role of the orexin system in stress response.

Neuropharmacology 154, 68-78. DOI: 10.1016/j.neuropharm.2018.09.034. 
Schwartz, M.D., Kilduff, T.S., 2015. The neurobiology of sleep and wakefulness. Psychiatr. Clin. North Am. 28, 615-644.

Sutcliffe, J.G., de Lecea, L., 2000. The hypocretins: Excitatory neuromodulatory peptides for multiple homeostatic systems, including sleep and feeding. J Neurosci. Res. 62, 161-168.

Thompson, M.D., Xhaard, H., Sakurai, T., Rainero, I., Kukkonen, J.P., 2014. OX 1 and $\mathrm{OX}_{2}$ orexin/hypocretin receptor pharmacogenetics. Front. Neurosci. 8, 57.

Tyree, S.M., Borniger, J.C., de Lecea, L., 2018. Hypocretin as a hub for arousal and motivation. Front. Neurosci. 9, 413.

Van Kampen, H.S., 2015. Violated expectancies: cause and function of exploration, fear, and aggression. Behav. Processes 117, 12-28.

Wiskerke, J., James, M.H., Aston-Jones, G., 2020. The orexin-1 receptor antagonist SB-334867 reduces motivation, but not inhibitory control, in a rat stop signal task. Brain Res. 1731, 146222. doi: 10.1016/j.brainres.2019.04.017 